\title{
Optic Disc and Macula Localization from Retinal Optical Coherence Tomography and Fundus Image
}

\author{
Rodiah $^{1}$, Sarifuddin Madenda ${ }^{2}$, Diana Tri Susetianigtias ${ }^{3}$, Dewi Agushinta Rahayu ${ }^{4}$, Ety Sutanty $^{5}$ \\ 1,3,4,5 Department of Informatics, Gunadarma University, Indonesia \\ ${ }^{2}$ Doctoral Program in Information Technology, Gunadarma University, Indonesia
}

\begin{tabular}{l} 
Article Info \\
\hline Article history: \\
Received Jul 22, 2018 \\
Revised Oct 3, 2018 \\
Accepted Oct 25, 2018 \\
\hline Keyword: \\
Fundus \\
Macula \\
OCT \\
Optic Disc \\
Retinal \\
\hline
\end{tabular}

\begin{abstract}
This research used images from Optical Coherence Tomography (OCT) examination as well as fundus images to localize the optical disc and macular layer of retina. The researchers utilized the OCT and fundus image to interpret the distance between macular center and optic disc in the image. The distance will express the area of macula that can be employed for further research. This distance could recognize the thickness of macula parameters diameter that will be used in localizing process of optic disc and macula. The parameters are the circle radius, the size of window's filter, the constant value and the size of optic disc element structure as well as the size of macula. The results of this study are expected to improve the accuracy of macula detection that experience the edema.
\end{abstract}

Copyright (C) 2018Institute of Advanced Engineering and Science. All rights reserved.

\section{Corresponding Author:}

Rodiah,

Department of Informatics,

Gunadarma University,

Margonda Raya Street 100, Pondok Cina, Depok, Indonesia.

Email: \{rodiah, sarif, diants, dewiar, ety_s\}@staff.gunadarma.ac.id

\section{INTRODUCTION}

One of the indicators for diabetic retinopathy in advance stage is edema macula. It is a leakage of fluids in retina. Diabetes can cause small blood vessel in this area to release fluid or fat. Edema macula may affect the patient's vision center and the worst-case edema macula may cause blindness [1]. Macula is a part of retina that allows eye to see soft details with a spherical morphology that located in the back of retina at $3.5 \mathrm{~mm}$ temporal distance and $0.5 \mathrm{~mm}$ smaller to the discus [2]. In the macula, there are vessels that play important role as photoreceptor. The study in sharpening the retinal fundus images was performed by the researchers using adaptive histogram and morphological operation of tophat as well as bothat. Blood vessel segmentation is necessary to perform in localizing other objects of retinal fundus images. These images are optic disc and macula [3]. In addition, retinal vascular extraction also performed using curvelet transformation and morphological operation that combine two operations. Those operations are tophat dan bothat [4]. The localization of the optic disc and macula area must be seperated from the blood vessel. Therefore, the sharpness of human vison is highly dependent on this section [5]. The macula lies in the midposterior of retina.

One of the initial phase in performing macula detection that experienced edema is by localizing the area of optic disc and macula area of retinal image. Optic disc localization in this study was carried on through tagging the connected component based on color intensity that have similarities with exudates by checking pixel (P) per pixel (from left to right side and from top to bottom direction) to identify the connected pixels area. The connected pixel area is an area from the bordered pixel that have the same intensity or intensity value in a set of $\mathrm{V}$ (in binary image $\mathrm{V}=\{1\}$ ). The connected component for input image follows the connectivity rules (4, 8 or m-connectivity) [6]. This study proposes a new technique to 
analyze the swelling of the macula. This study consists of two stages. The first stage is vessel extraction and the second one is vessel reconstruction.

The image input is a fundus green channel image that is converted into grayscale image. In this study, Speeded Up Robust Features (SURF) algorithm was used to describe the process of macula detection on retinal image by discovering the compatibility between the features points of each image. This study also used Random Sample and Consensus (RANSAC) algorithm to match the points that correspondent and Gaussian function algorithm for smoothing the macular image splicing. The RANSAC algorithm that was implemented in this study used to select random subset from original image to achieve the best results in localizing the swollen macula area [7]. This study determined the presence of macula edema by detecting the presence of fovea. Fovea lies in the middle of macula. Fovea and optic disc detection were performed using motion pattern recognition. The feature selection was carried on using Principal Component Analysis (PCA) by creating new feature derived from extraction of linear features combinations. Therefore, they can be used to reduce the selected feature dimension which is edema macula. Gaussian derivative also used in this study to capture the characteristic of edema macula patters that will be detected [8].

The retinal fundus optic disc locatization was performed in several steps. The first step was performing green chanel image fundus extraction. The next step was carry out fitur optic nerve head $(\mathrm{ONH})$. ONH was performed based on the contour from optic disc cup area. In performing the extraction, the researchers converting the image to grayscale and executing thresholding with dilation and erotion operation. The next step is performing edge detection using dilation operation to create matrix feature. The result of matrix feature will be used as an input for training and testing process. The training and testing process in this study using SVM method [9]. The research was performed by establishing an automatic diagnostic system to detect macule using fluorescein image. In the preliminary stages, noise reduction was done in preprocessing phase to eliminate the object details beside the macula and to maintain its lightning distribution with Gaussian filter. There were several processes in this study, for instance blood vessel segmentation with Gaussian filter, optic disc detection, and red lesion detection to segment micro aneurysm feature.

The segmented feature will describe the shape, color and contrast of the detected candidates [10]. The optic disc detection in this study was done by detecting the maximum area with the highest pixel value. Then the retinal fundus image along with the optic disc that already being detected will perform local image features process. Pixel weighting was carried out to gain neighboring influence in performing segmentation by clustering each pixel inside the cluster center. The macula classification phase proposed in this study is based on some parameters such as the size of macula window's area and spatial relationship between pixels in the retinal fundus [11].

The medical examination of the edematous macules is done by direct observation by the physician through Optical Coherence Tomography (OCT) examination. This examination will provide cross sectional of retinal issue images. In this examination, the thickness of the retina and macula will be measured, which indicate the occurrence of swelling and fluid clots in the area. Determination of optic disc localization in this study carried on using fundus image. Disc localization and macula are principal factors that determined the distance interpretations between the centers of macula with optic disc in pixel representation to know the diameter of macular thickening. This study used some parameters. For instance, the circle radius, the size of window filter, and constant value as well as the size of optic disc structuring element and macula. The result of this study is expected to improve the accuracy of macular detection that experienced edema.

\section{RESEARCH METHOD}

\subsection{Data Sets}

This study used imaging images with Optical Coherence Tomography (OCT) and Fluorescein Angiography fundus image as can be seen in Figure 1.

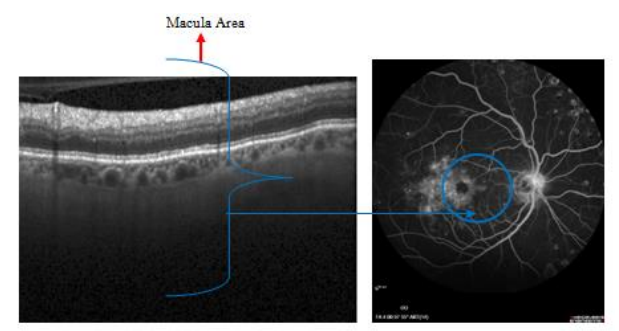

Figure 1. OCT and Fundus Image 
In the fundus retinal image in Figure 1, the image shows quite low contrast of retina's macula. Macula objects are sometime hindered by another object that indicate retinal abnormalities such as the exudate, dot and blood hemorrhage, micro aneurysm as well as Intra-Retinal Microvascular Abnormalities (IRMA's). These abnormalities can cause difficulties and in localization of macular area.

\subsection{Estimate Optic Disc Position}

In Fluorescein Angiography (FA) fundus image, the initial phases of the process were detecting the center estimation and the diameter of optic disc. The estimation of macula area was based on the distance and its position with optic disc. This distance is relatively constant in each retina. The size of the disc on each retina image of the patient is varies, but generally the size of optic disc has diameter between $1.8 \pm 2 \mathrm{~mm}$ inside the image fundus with standard size of 768 x 576 pixel. There are some factors that make optic disc edge detection accurate. Some parts of the optic disc will be obscured by many retinal blood vessels. In general, the process in localizing the existence of the optic disc in this study was performed by the following algorithm:

1. Green channel FA fundus image extraction. The green channel was selected as the primary color since green color has the high intensity compare to the others two colors; red and blue in an image with RGB) color as can be seen in Figure 2. Below is the mathematical formula to find the green channel selection :

$$
g=\frac{G}{(R+G+B)}
$$

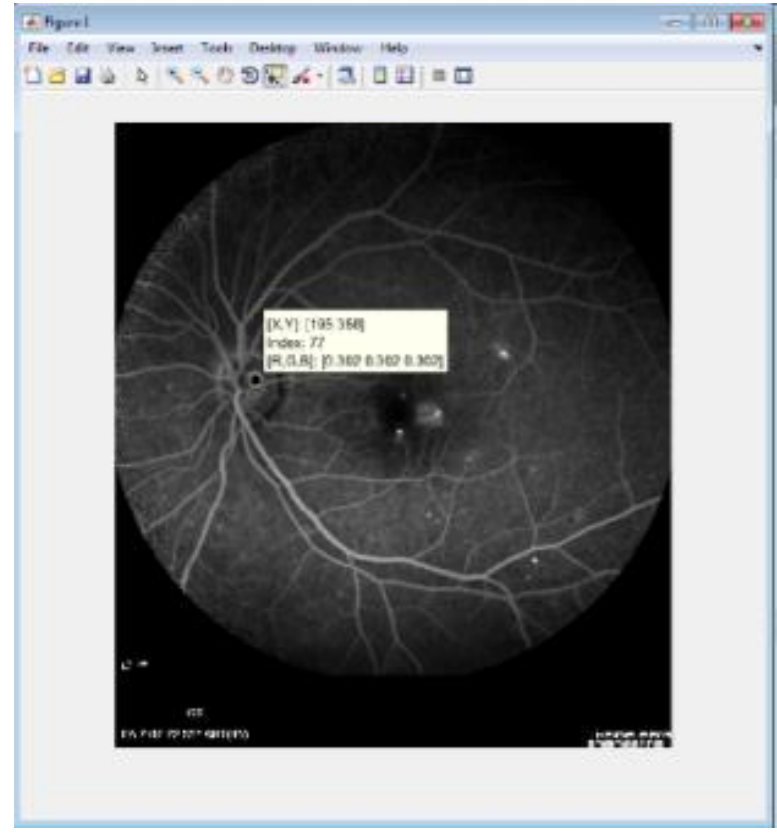

Figure 2. OCT and Green Channel Fundus Fluorescein Angiography (FA) Image

\subsection{Morfology}

Formula (1) is performed to find a green channel in an image, while R, G and B are stand for Red, Green and Blue sequentially. In green channel, the detail of image can be seen clearly and comprehensively. Whereas the used of red channel only going to show the image edge while in blue channel merely provide just part of the image's detail and contain a lot of noise. Based on image in Figure 2, the contrast of optic disc can be observed better in green channel compared to red and blue channel in RGB image. Therefore, this study only used green channel image to compute the optimal threshold. Figure 3 shows the green channel fundus Fluorescein Angiography (FA) image and its histogram. It can be seen that the pixel that corresponding with optic disc have higher intensity in histogram. 


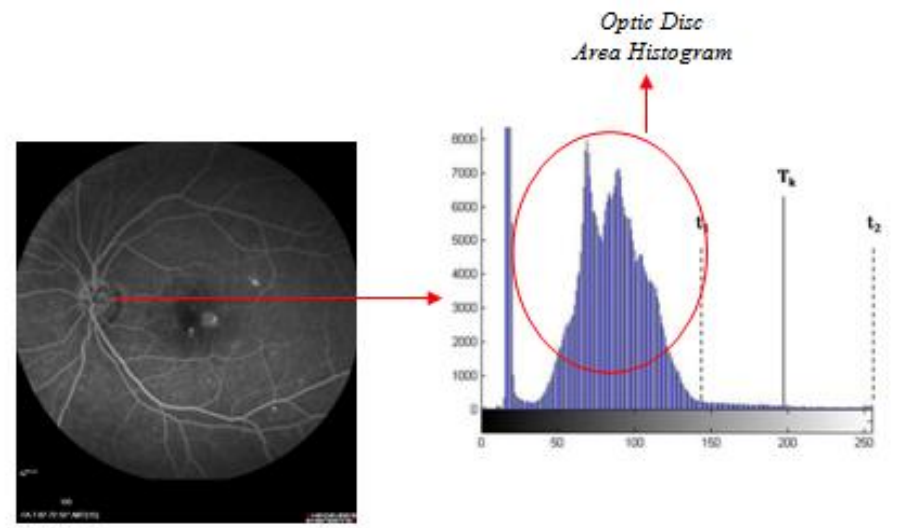

Figure 3. OCT and Green Channel Fundus Fluorescein Angiography (FA) Image

2. The localization of optic disc position. Optic disc localization can be performed by considering the geometry shape of optic disc on several connected components. The difficulties in estimating the optic disc position is the presence of other objects outside the optic disc image that has connected component as well as can be seen in Figure 4.

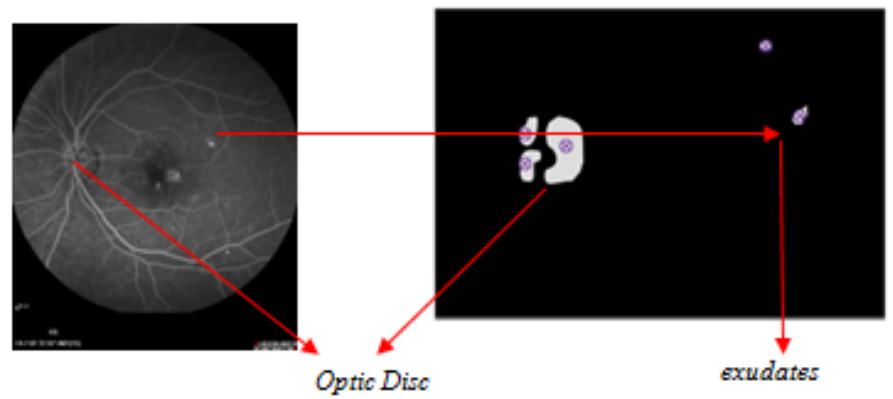

Figure 4. Connected Component Detection on Optic Disc

3. Each component that connected to the image will be labelled with the number of pixels in the component. Spatial coordinate of each connected component is calculated. Component is assumed to have maximum number of pixels with maximum diameter of optic disc which is $2 \mathrm{~mm}$. Consequently, if one component of the image spatial coordinate is within 50 distance pixels from spatial coordinate of the mean largest component, the object will be combined and the new mean of image spatial coordinate will be computed as can be seen in Figure 5.

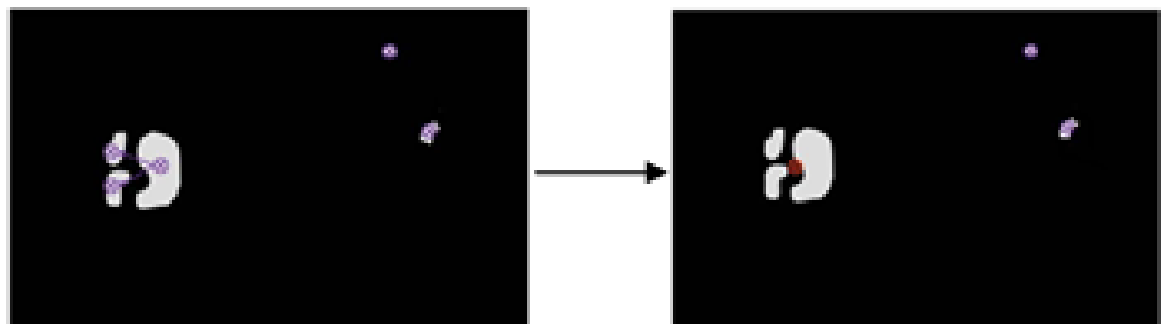

Figure 5. Position Estimation of Optic Disc

4. Performing connected component tagging with object geometry approach using some parameters below :

Optic Disc and Macula Localization from Retinal Optical Coherence Tomography and Fundus ... (Rodiah) 
1) The value area is computed based on the maximum and mean intensity in the optic disc area.

2) Restricting the area that are detected as an optic disc. This area is the number of image dimension with angle and width parameters in retinal image that is written in an array form using the formula:

BoundingBox $=[$ corner width $]$

3) Retinal optic disc position estimation based on the ratio where the shape of the optic disc is circle. In a circle object the properties that can be discovered are [12]: Radius of the circle $r$, circle diameter $d$, wide of circle $L$, circumference of circle and $P$ parameter. The formula to localize optic disc position based on the ratio of round

4) The extensive and parameter value as the property of this circle can be performed in optic disc regions which will be localized as the basic form of the roundabout size using this formula:

Ratio $=\frac{P^{2}}{4 \pi L}$

5) If the result value of detected object ratio yields 1 and has the intensity equal or almost the same as optic disc intensity, this detected object will be marked (localization). The objects that have different characteristic with the optic disc will not be marked. In general, the optic disc illustration can be seen in Figure 6.

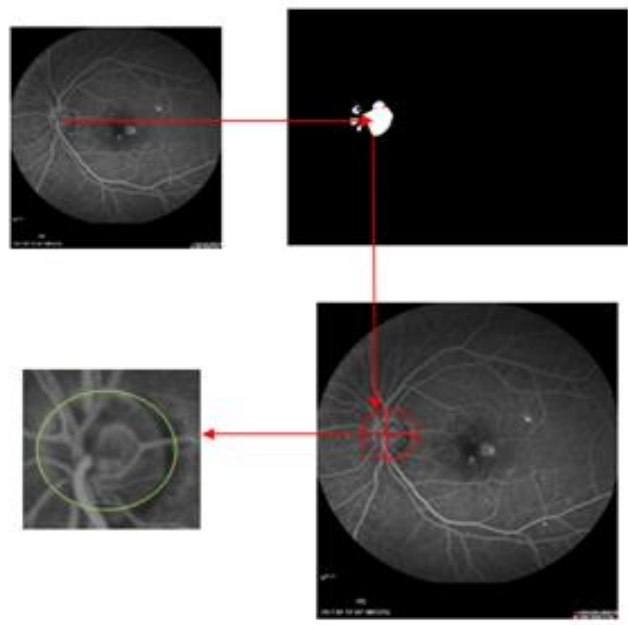

Figure 6. Optic Disc Position Localization Illustration

The ratio value of 1 is based on the ratio length of major axis and minor axis of the object that was detected in fundus image as can be seen in Figure 7.

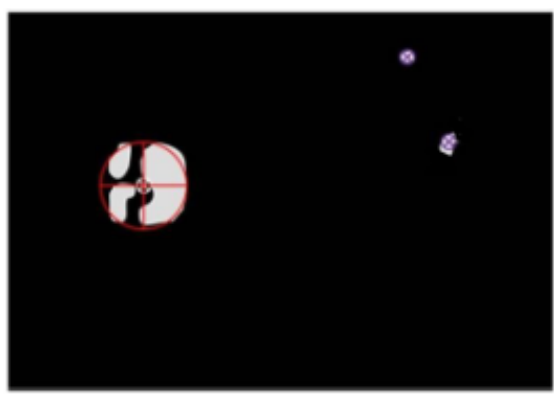

Figure 7. Ma Detected Optic Disc 


\subsection{Localization OCT Macula Layers}

Localization of macular image layer area was performed on preprocessing phase. This localization was carried on in calculating the macular area outside the normal size of retina macular image. The macula area has the thickness of $400 \mu \mathrm{m}$, diminishing on fovea with the size of $150 \mu \mathrm{m}$, and becoming thinner on ora serrata with the thickness of $80 \mu \mathrm{m}$ [13]. The macula has very low contrast inside the retinal image and sometime the area of macula is blocked by the exudates or the bleeding in the retina's region [14]. The algorithms of preprocessing phase on OCT macular images area can be explained below:

1. Read the OCT Image

2. Performing the blurring process to reduce the noise in OCT image. The intensity value of the image changes a lot as in Figure 8. The noise in OCT images have the form of dots or pixels with different intensity. This study used the first derivative of Gaussian filter to reduce the noise on OCT image with the formula below:

$$
G(x, y)=\frac{1}{2 \pi \sigma^{2}} e^{-\frac{x^{2}+y^{2}}{2 \sigma^{2}}}
$$

Where $\sigma$ denote the derivative standard from pixel distribution of OCT image OCT $I(x, y)$. Assumed a distribution have an average value equal to $0(\sigma=1)$.
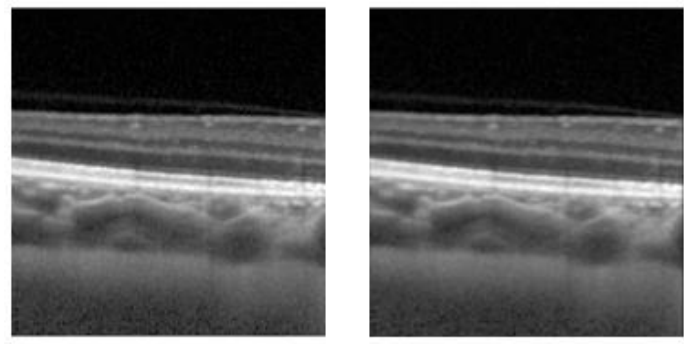

Figure 8. Noise Reduction Process and OCT Macula Image

(a) Original Image (b) Image from Blurring Process

3. Performing edge detection on image gradient at horizontal direction from the filtering image using the algorithms below:

a) Read the image resulted from blurring phase.

b) Read size of the image with function $[\mathrm{N}, \mathrm{M}]=\operatorname{size}(\mathrm{G}) .[\mathrm{N} \mathrm{M}]$ is a pixel line and column of an image obtained from blurring phase $G(x, y)$. The first derivative of image function $\mathrm{G}(x, y)$ towards $x$ and $y$ axis that are computed using formula [15] :

$$
\frac{\partial G}{\partial x}(x, y) \text { and } \frac{\partial G}{\partial y}(x, y)
$$

Whereas, the vector gradient and the amplitude can be calculated with [9] :

$$
\begin{aligned}
& \nabla G(x, y)=\left[\begin{array}{l}
\frac{\partial G}{\partial x}(x, y) \\
\frac{\partial G}{\partial y}(x, y)
\end{array}\right] \\
& |\nabla G|(x, y)=\sqrt{\left(\frac{\partial G}{\partial x}\right)(x, y)^{2}+\left(\frac{\partial G}{\partial y}\right)(x, y)^{2}}
\end{aligned}
$$

4. Referring to equation 7, the first partial derivative of images from blurring phase were computed based on the two-closed neighboring pixel. Therefore, the gradient can be obtained: 


$$
\nabla G(x, y)_{x}=\frac{\partial G}{\partial x}(x, y)=G(x+1, y)-G(x, y)
$$

$\nabla G(x, y)_{x}$ is an image gradient on $x$ horizontal direction that produced edge of object in the shape of horizontal line, as in Figure 9.

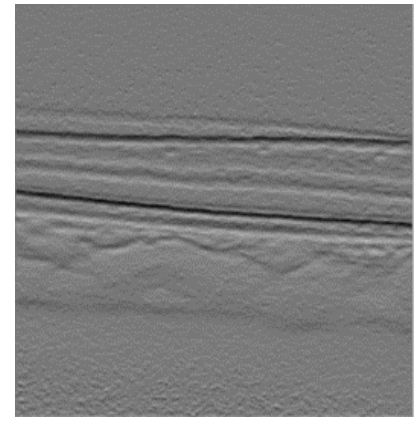

Figure 9. Image Gradient on Horizontal Direction

5. Labeled the connected component that link horizontal image gradient with adjacent matrix. $x$ pixel is said to be adjacent with $y$ pixel if there is a connected connection between them. Two images subset $S_{1}$ and $S_{2}$ are adjacent if some pixels on $S_{1}$ adjacent with some $S_{1}$ pixels as in Figure 10.

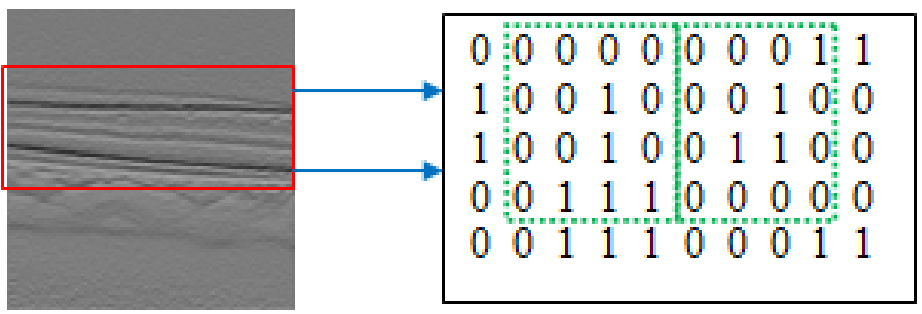

Figure 10. Subset $S_{1}$ and $S_{2}$

Path from pixel $x$ with coordinate $(p, q)$ for $y$ pixel with coordinate $(r, s)$ is a pixel order that differ from coordinate $\left(p_{o}, q_{0}\right),\left(p_{1}, q_{1}\right), \ldots\left(p_{n}, q_{n}\right)$

where:

$$
\begin{aligned}
& n=\text { Path } \\
& \left(p_{0}, q_{0}\right)=(p, q),\left(p_{n}, q_{n}\right)=(r, s) \\
& \left(p_{1}, y_{1}\right) \text { is adjacent where : } \\
& p_{i-1}, q_{i-1}
\end{aligned}
$$

6. Compute the distance between $x(p, q)$ and $y(r, s)$ using city block distance with 4 connectivity as can be seen in Figure 11 using the formula :

$$
D_{4}(x, y)=|p-r|+|q-s|
$$




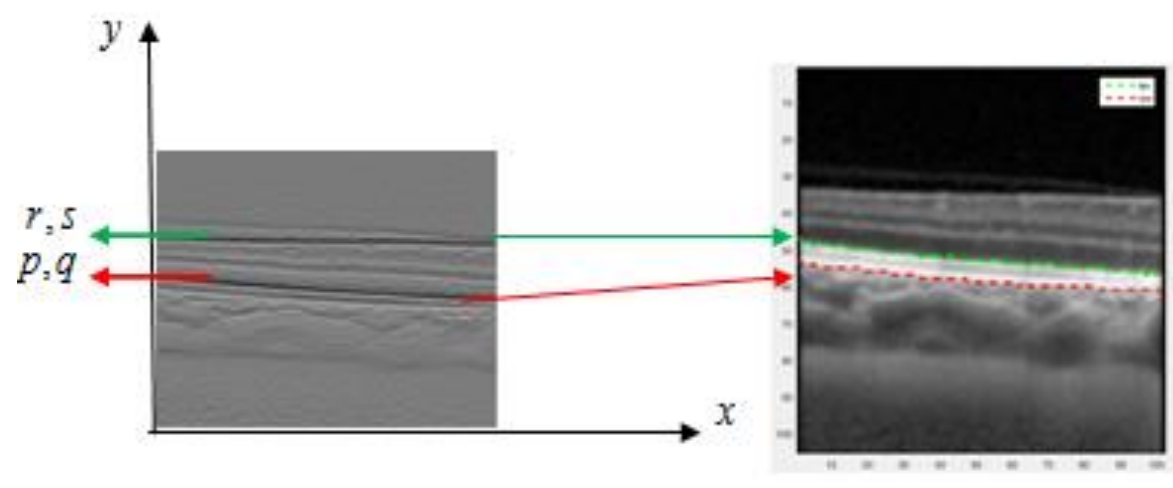

Figure 11. City Block Distance with 4 Connectivity

\section{RESULTS AND ANALYSIS}

\subsection{Experimental Image}

The localization of the optical disc position was performed in geometric optic disc form by considering several connected components. The difficulty in performing the optic disc position estimation was the occurrence of another object outside the optical disc that has a connected component as well. Figure 12 shows the proposed algorithm in this study succeed to localize the presence of optic disc on fundus image.

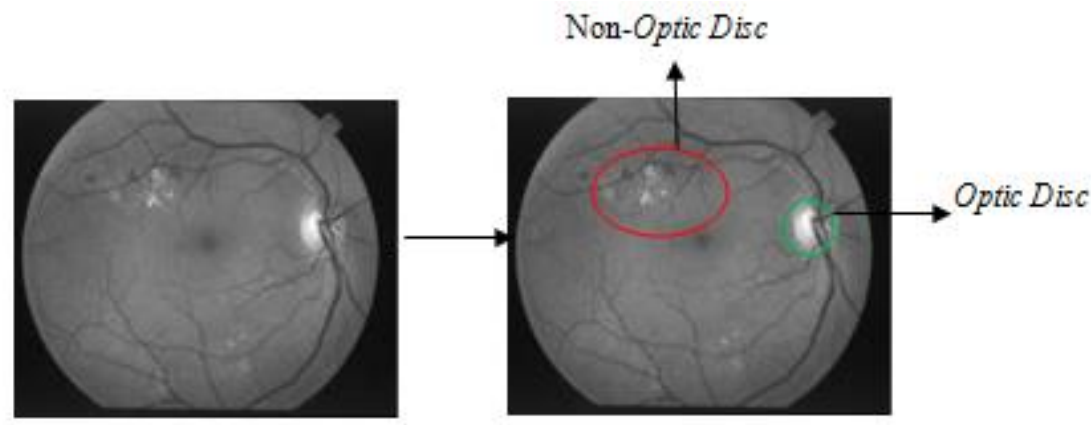

Figure 12. Estimation Image OD Position

In previous study, the process of retinal fundus optic disc images localization was performed using combination of morphological operation and optic disc center point determination. This method was carried out by detecting the geometric shape in the parametric form, where the shape of the optic disc is circular. The exploitation of Hough Transformation [16] was carried out to search the most suitable geometric form that correspond to the set of points in the image. The transformation is done to find the lines and the curves that passing by many points inside the image. These lines and curves are the one that near and most correspond with the data in the image matrix. The selection of the circular radius greatly affect the accuracy of the area localization of optic disc. If the circular parameter value is too small or too big, it will affect the distance of macula's center from the central of optic disc, resulting macula ROI will be unsuitable. The study in this paper used roundness ratio. This roundness ratio is the ratio of the radius at the smallest angle to the radius average of fundus image as object. The ratio of this study was proposed by the researchers to perform comparison between the shorthest axis with the longest axis of the optic disc. Table 1 shows examples of FA fundus Image OD estimation position.

As can be seen on Table 1, the optic disc with the roundness object ratio approach succesfully localizing its position correctly. This ratio is the average geometric diameter of optic disc with its longest diameter, where the object with a rounded geometric shape connected component in fundus image is an optic disc. Therefore, those area are localized as optic disc. 
Table 1. Optic Disc Localization Result

\begin{tabular}{cccc}
\hline Image & FA Fundus Image & Optic Disc Localization Result \\
\hline FA1 & \\
FA3 & \\
FA4 & \\
FA5
\end{tabular}

\subsection{OCT Macula Image Localization}

The macula's layers play important role in human's vision. These multiple layers are located in three different areas (Fovea, Avascular Zone and Foveolar) as can be seen in Figure 13.

(a)

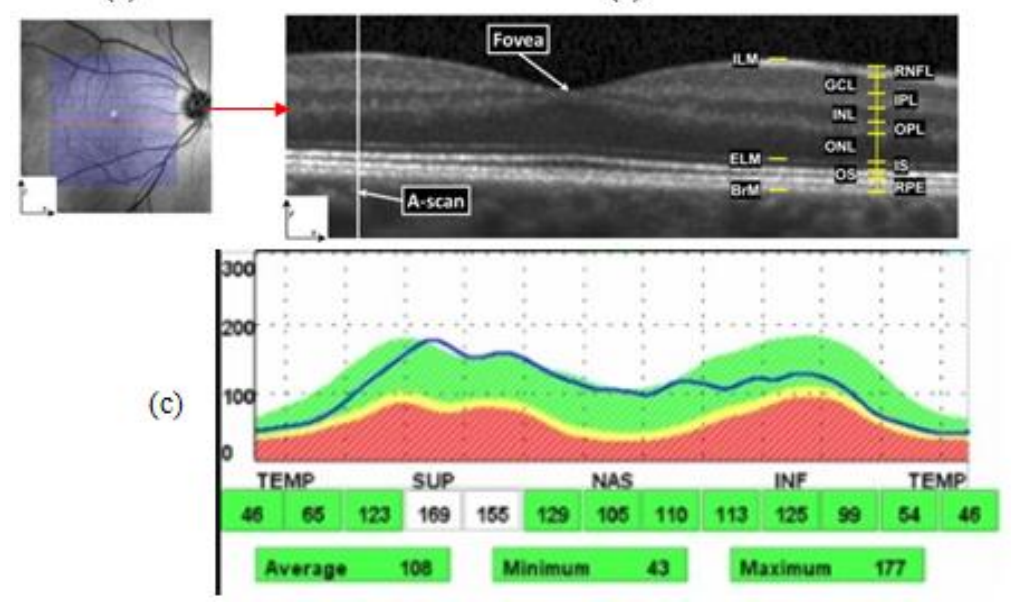

Figure 13. (a) Fundus Scanned Image FA, (b). Area and Layers of OCT Macula, (c). Macula Area Position

Edema macula is associated with vision blindness in non proliferative diabetic retinopathy. The symptoms are characterized by (i) the retinal thickening at or within $500 \mu \mathrm{m}$ distance from the center of macula, (ii) the occurrence of exudates or within $500 \mu \mathrm{m}$ distance from the center of macula followed by the thickening of the retinal surround the macula's center and (iii) retinal thickening that greater than one disc diameter (DD) distance from the center of macula. The changes inside the macula's layer area will influence the peripheral's vision. Therefore, it is very important to localize the area of macula in detecting macula that has edema [17]. Figure 14 shows the example of OCT macula layers localization's results on Inner Limiting Membrane (ILM) area and RPE OCT macula image. 


\begin{tabular}{cc}
\hline Code & Note \\
\hline ILM & Inner Limiting Membrane \\
NFL/ GCL & Nerve Fiber Layer \\
IPL/ INL & Inner Plexiform Layer \\
INL/ OPL & Inner Nuclear Layer \\
OPL/ ONL & Outer Plexiform Layer \\
OPL/ ONL & Outer Nuclear Layer \\
IS/ OS & Inner Segment \\
RPE & Retinal Pigmented Epithellium \\
\hline
\end{tabular}

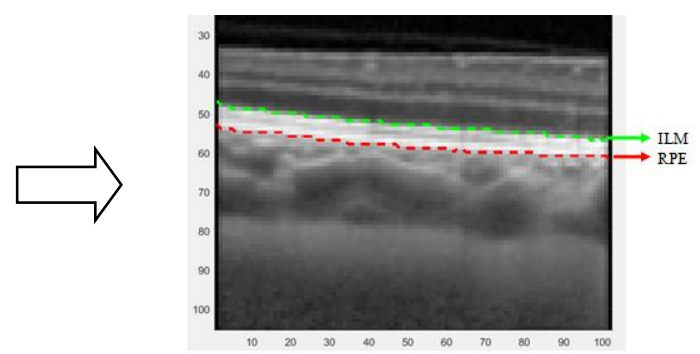

Figure 14. Macula Area Localization and OCT Macula Layer [17]

Many approaches had been done by some researchers in OCT macula images localization. This localization was carried out through selecting the pixel that will be used as seed point and by searching the similar neighboring pixels. The pixels area then expanded by adding similar pixel to the neighboring pixels. Unfortunatelly, researchers facing difficulties of performing segmentation on OCT makula area. The difficulties are on color similarities and on texture of each macula layer. Macula layers consist of 8 layers OCT. In this study, the researchers proposed the utilizing of adjacency matrix to present the pixels of macular layer. These layers are closed to the other macula's layer. The adjacency was performed by presenting pixel 1 which indicating if node $\mathrm{i}$ to node $\mathrm{j}$ has an edge and 0 otherwise (when the node has no edge).

\section{CONCLUSION}

The proposed algorithm succeeds to localize the existence of optic disc. This optic disc is based on the connected component that distinguished optic disc with the other object of the image fundus such as the exudates. The result of macula's area localization on OCT image can compute the macula area outside the normal size of macula retina image. The approach with geometric optic disc ratio succeeds to localized the optic disc. The exploitation of adjacendy matrix able to distinguish every layer of OCT macula image despite their similarities and color neighboring.

For the further research, the thickness of 7 layers of macula OCT image will be detected. This thickness will be used as reference to detect the sign of the presence of edema macula on OCT image.

\section{ACKNOWLEDGEMENTS}

We are indebted to the experts who have contributed towards development of the template. The authors would like to acknowledge to Gunadarma University.

\section{REFERENCES}

[1] Bowling, Kanskis's Clinical Ophthalmology A Systematic Approach, Eighth Edition, ISBN: 978-0-7020-5572-0, Elsevier, Sydney, New South Wales, Australia. (2016)

[2] Vaughan, Asbury, Riordan Eva. General Ophthalmology. Edition 17. London: McGraw-Hill. 2007.

[3] Razieh Akhavan, Karim Faez, "A Novel Retinal Blood Vessel Segmentation Algorithm using Fuzzy Segmentation", International Journal of Electrical and Computer Engineering (IJECE), Vol. 4, No, 2016.

[4] K. Gayathri, D. Narmadha, K. Thilagavathi, K. Pavithra, M. Pradeepa, "Retinal Blood Vessels Extraction Based on Curvelet Transform and by Combining Bothat and Tophat Morphology", International Journal of Electrical and Computer Engineering (IJECE), Vol. 4, No. 3, 2014.

[5] Liesegang, Skuta, Cantor. Retina and Vitreous. Basic and Clinical Course. Section 12. San Fransisco, California: American Academy of Ophthalmology. 2004.

[6] Susetianingtias, D.T., Madenda, S., Rahayu, D.A., Rodiah, "Retinal microaneursym detection using maximally stable external region algorithm", International Journal on Advanced Science, Engineering and Information Technology, vol 6 No. 5, 2016.

[7] Giancard, Karnowski, Grisan, Favaro, Ruggeri, and Chaum. "Textureless Macula Swelling Detection with Multiple Retinal Fundus Images". IEEE Transactions on Biomedical Engineering, Vol. 58, No. 3. (2011)

[8] Fowjiya, Karnan, Sivakumar. "An Automatic Detection and Assessment of Diabetic Macular Edema Along with Fovea Detection from Color Retinal Images", International Journal of Computer Trends and Technology (IJCTT). Volume 4 Issue 4. 2013.

[9] Anindita Septianirini, Hamdani, Dyna Marisa Khairina, "The Contour Extraction of Cup in Fundus Images for Glaucoma Detection", International Journal of Electrical and Computer Engineering (IJECE), Vol. 6, No. 6, 2016.

[10] Devashree. Zinjarde, Mohota. "Computer Aided Diagnosis of Macular Edema Using Color Fundus Images: A Review”. Int. Journal of Engineering Research and Applications. ISSN: 2248-9622, Vol. 4, Issue 3. 2014. 
[11] Rahim, Palade, Jayne, Holzinger and James Shuttleworth. "Detection of Diabetic Retinopathy and Maculopathy in Eye Fundus Images Using Fuzzy Image Processing". International Conference on Brain Informatics and Health BIH 2015: Brain Informatics and Health, pp 379-388. 2015.

[12] Peter S. "Measuring objects, Computer Vision and Math contains: mathematics courses, covers: image analysis and data analysis, provides: image analysis software article". Available From : http://inperc.com/. (2011)

[13] Hilldebrand and Fielder. "Anatomy and Physiology of the retina. Pediatric Retina", 39. DOI: 10.1007/978-3-64212041-1_2. Springer-Verlag Berlin Heidelberg. (2011)

[14] Susetianingtias D.T., Madenda S., Rodiah. "An Approach to Exudates Detection using Color Reference Segmentation in Retinal Fundus Image”. International Journal of Computer Applications. Volume 146, Number 2. 2016.

[15] Madenda S. "Pengolahan Citra \& Video Digital: Teori, Aplikasi dan Pemrograman Menggunakan MATLAB", Penerbit Erlangga. 2015.

[16] Handayani Tjandrasa, Ari Wijayanti, Nanik Suciati, "Optic Nerve Head Segmentation Using Hough Transform and Active Contours", TELKOMNIKA (Telecommunication Computing Electronics and Control), Vol.10, No.3. (2012)

[17] Quillen D.A., Blodi B.A., and Bennett T.J. "Clinical Features of Retinal Disease". Quillen DA and Blodi BA, eds. Clinical Retina. Chicago: AMA Press, 2002.

\section{BIOGRAPHIES OF AUTHORS}

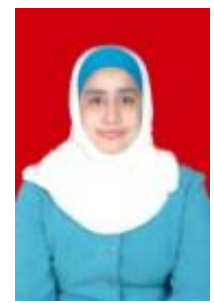

Rodiah currently active as Vice Head of Postgraduate Academic System Development and a lecturer at Gunadarma University. Research interest is in medical image processing.

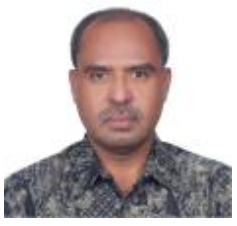

Sarifuddin Madenda is a professor. Currently he is active as the Head of PhD Programs of Information Technology and a lecturer at PhD Program at Gunadarma University. Her Research interest is in signal, video and image processing.

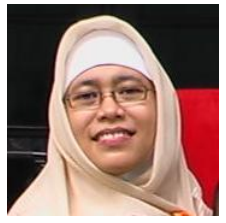

Dewi Agushinta Rahayu currently active as Vice Dean for Academic Affair and a lecturer at Gunadarma University. Research interest is in face in recognition system.

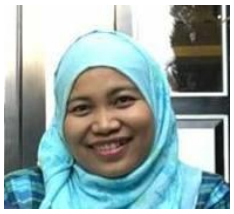

Diana Tri Susetianingtias currently active as a lecturer at Gunadarma University. Research interest is in online medical image processing

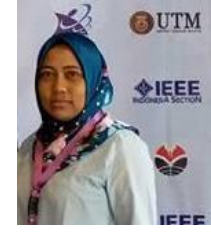

Ety Sutanty is a Doctoral student at Gunadarma University. Her Research interest is in Image Processing 